\title{
Liapunov theory applied to control, games, and boundedness of generalized dynamical systems
}

\author{
Russel James Stonier
}

Since its introduction, Liapunov's qualitative direct or "second" method has been applied in a variety of problems, particularly in nonlinear mechanics and control systems.

In Chapter 1, we apply the Liapunov method in a study of various boundedness and ultimate boundedness properties of generalized dynamical systems. Strong and weak reachability of compact sets by such systems is also discussed.

We proceed in Chapters 2,3 , and 4 to examine the application of Liapunov theory in the discussion of qualitative objectives of two person games whose dynamics are firstly modelled by contingent equations, and then more abstractly by generalized dynamical systems. Particular attention is given to the modelling of two person games by generalized dynamical systems (Chapters 3 and 4 ), and the qualitative objectives are so chosen as to make use of some results established in Chapter 1 .

In Chapter 5, we again employ the Liapunov method in finding sufficient conditions in a control problem. Sufficient conditions are found for the qualitative objective - reachability of the target set (controllability); and for the quantitative objective - minimization of an integral performance index (optimal control). For problems with particular performance index, combined qualitative and optimal sufficiency theorems are given for both open loop and closed loop control functions. Our

Received 3 July 1978. Thesis submitted to the University of Queensland, March 1978. Degree approved, September 1978. Supervisor: Dr J.M. Skowronski. 
results are extended to the saddle point problem in a two person differential game. 